\title{
Molecular Identification of Taenia hydatigena from Sheep in Khartoum, Sudan
}

\author{
Rosline James Muku', Hong-Bin Yan', John Asekhaen Ohiolei', Abubakar Ahmed Saaid², Sara Ahmed', \\ Wan-Zhong Jia', Bao-Quan Fu',* \\ 'State Key Laboratory of Veterinary Etiological Biology, Key Laboratory of Veterinary Parasitology of Gansu Province, Lanzhou Veterinary Research \\ Institute, Chinese Academy of Agricultural Sciences, Lanzhou 730046, P.R. China; ${ }^{2}$ Central Veterinary Research Laboratory, Animal Resources \\ Research Corporation, P.O. Box 8067 El-Amarat, Khartoum, Sudan
}

\begin{abstract}
The cestode Taenia hydatigena uses canids, primarily dogs, as definitive hosts, while the metacestode larval stage cysticercus infects a range of intermediate hosts, including domestic animals such as goats, sheep, and pigs. Cysticercosis due to T. hydatigena has large veterinary and economic drawbacks. Like other taeniids, e.g., Echinococcus, intraspecific variation is found among the members of the genus Taenia. In Africa, few studies are available on the epidemiology and distribution of $T$. hydatigena, and even fewer studies are available on its genetic variation. In this study, we molecularly identified 11 cysticerci from sheep in Sudan and demonstrated the genetic variation based on the NADH dehydrogenase subunit 1 (nad1) and cytochrome c oxidase subunit 1 (cox1) mitochondrial genes. The isolates were correctly identified as T. hydatigena with more than $99 \%$ similarity to those in the GenBank database. Low diversity indices and insignificant neutrality indices were observed, with 3 and 2 haplotypes for the nad 1 and cox 1 genes, respectively. The results suggest the presence of unique T. hydatigena haplotypes in Sudan, as haplotypes with $100 \%$ similarity were not found in the GenBank database. With few available studies on the genetic variation of $T$. hydatigena in Africa, this report represents the first insights into the genetic variation of $T$. hydatigena in Sudan and constitutes useful data.
\end{abstract}

Key words: Taenia hydatigena, genetic variation, cysticercus, Khartoum

The cestode Taenia hydatigena uses canids, primarily dogs, as definitive hosts, while the metacestode larval stage infects a range of intermediate hosts, including goats, sheep, and pigs. Cysticercosis due to T. hydatigena is of veterinary and economic importance, especially in small ruminants, where it causes production loss through clinical disease or damage to infected organs [1-3].

In Africa, a few studies are available on the epidemiology and distribution of T. hydatigena [4]. Prevalence reports, where available, have demonstrated high infection rates in sheep and goats and low infection rates in pigs [4-8]. In Sudan, data on the prevalence of $T$. hydatigena in livestock are scarce. However, a study conducted by El Badawi et al. [9] reported a high prevalence of T. hydatigena in sheep (32.4\%) and goats (29.0\%) and emphasized its economic importance and implications

\footnotetext{
- Received 28 March 2019, revised 26 November 2019, accepted 6 December 2019.

*Corresponding author (fubaoquan@caas.cn)

(c) 2020, Korean Society for Parasitology and Tropical Medicine

This is an Open Access article distributed under the terms of the Creative Commons Attribution Non-Commercial License (https://creativecommons.org/licenses/by-nc/4.0) which permits unrestricted non-commercial use, distribution, and reproduction in any medium, provided the original work is properly cited.
}

for livestock production.

Genetic variation has been well studied in most cestodes species, e.g., Echinococcus granulosus [10,11], and correlates with parasite morphology, host infectivity, drug and vaccine development, and ultimately control. Similarly, mitochondrial genome studies of Taenia spp. have also confirmed the existence of genetic variation within species [13,14], and in some cases, such variation has been suggested to correlate with pathological differences in different hosts $[15,16]$. The molecular epidemiology and genetic variation of T. hydatigena in Africa are underinvestigated; however, a few studies in countries such as $\mathrm{Ni}$ geria, Egypt and Tanzania have demonstrated nucleotide variation between isolates from sheep, goats, and pigs $[4,17,18]$. Meanwhile, studies describing the genetic population structure of T. hydatigena in Sudan and how they differ from other geographical regions, as well as the epidemiological significance, are lacking. Therefore, the aim of this study was to molecularly identify T. hydatigena of Sudanese origin and to investigate possible genetic variation based on the NADH dehydrogenase subunit 1 (nad1) and cytochrome c oxidase subunit 1 (cox1) mitochondrial genes. 


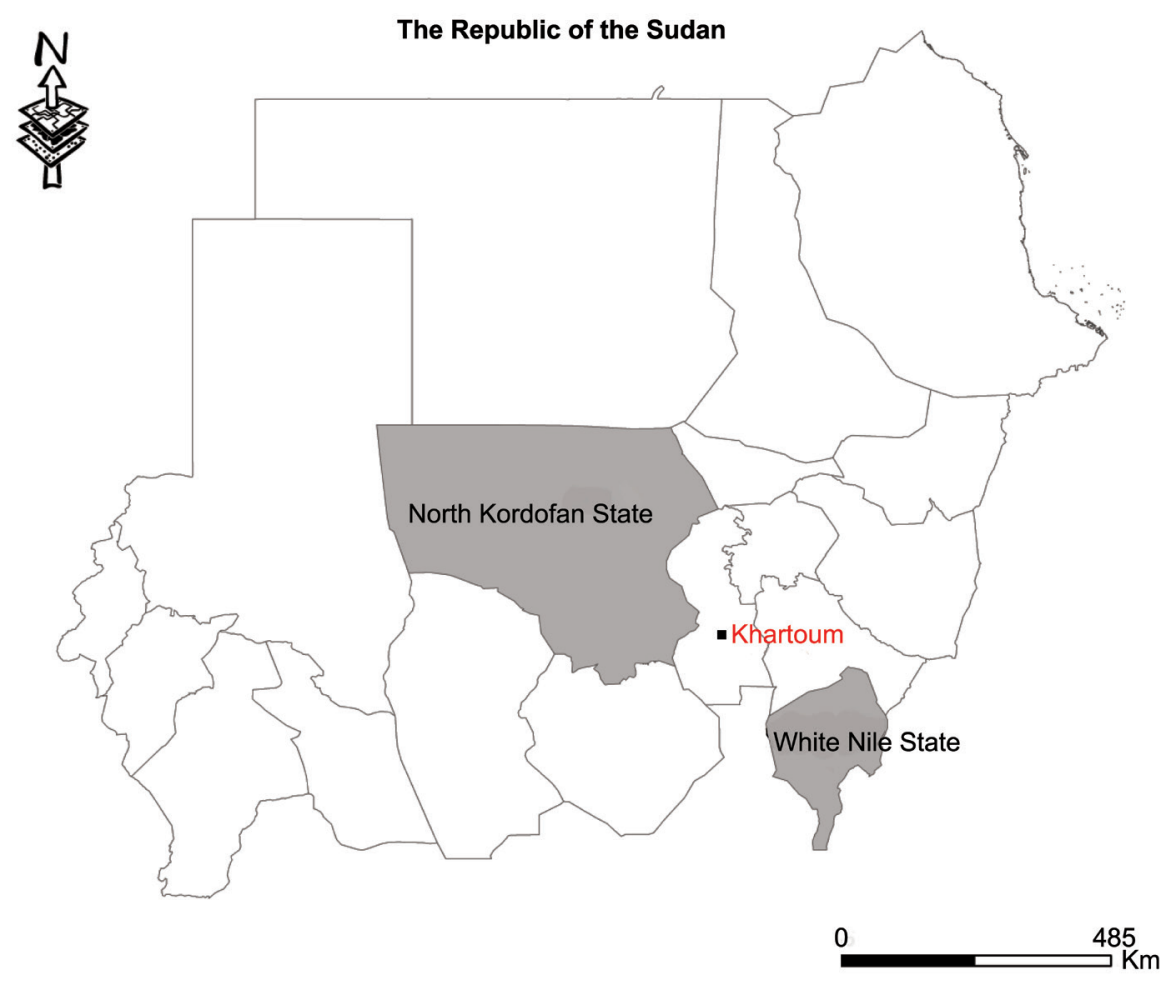

Fig. 1. Map showing study areas. Black dot, region where sheep were slaughtered. Grey region, sheep origin.

Thirty cyst samples $(2-5 \mathrm{~cm}$ in size) from liver were harvested from slaughtered sheep in various homes in the Western Nile (Al-Hajyosif and Al Gouz)- Khartoum, Sudan, during a meat/slaughtering inspection for the Addha festival (September 2017). A total of 1,500 sheep were examined during the inspection. However, the slaughtered sheep were said to have originated from North Kordofan State and the west White Nile State of Sudan (Fig. 1). DNA was extracted from a portion of each cysticercus using the phenol-chloroform extraction method. Extracted genomic DNA samples were stored at $-20^{\circ} \mathrm{C}$ until use. PCR amplification of the mitochondrial nad 1 and $c o x 1$ genes was performed using the following primer pairs specific for cestode parasites: forward 5'CARTTTCGTAAGGGBCCWAAWAAGGT and reverse 5'-CCAATTTCYTGAAGTTAACAGCATCA [19]; and a newly designed forward (5'-AGTCCTGATGCTTTTGGGTTCTATGGA-3') and a previously reported reverse primer (5'-AAGCATGATGCAAAAGGCAAATAAACC-3') [20], for the nad1 and cox 1 genes, respectively, with expected fragment sizes of 871 and 939 bp, respectively. PCR was conducted in a $25 \mu \mathrm{l}$ reaction mixture containing $12.5 \mu \mathrm{l}$ Premix Ex Taq $^{\mathrm{TM}}$ version 2.0 (Takara Bio, Japan), $10 \mathrm{pmol}$ of each primer, $0.5 \mu \mathrm{l}$ of genomic DNA extract (20-200 ng), and
RNAse free water up to the final concentration of $25 \mu$. The reaction was performed under the following conditions: initial denaturation at $95^{\circ} \mathrm{C}$ for $5 \mathrm{~min}, 35$ cycles of denaturation at $95^{\circ} \mathrm{C}$ for $30 \mathrm{sec}$, annealing at $55^{\circ} \mathrm{C}$ for $30 \mathrm{sec}$ and elongation at $72^{\circ} \mathrm{C}$ for $60 \mathrm{sec}$, and a final extension at $72^{\circ} \mathrm{C}$ for $10 \mathrm{~min}$. Then, $5 \mu \mathrm{l}$ of the PCR products were analyzed in a $1.5 \%(\mathrm{w} / \mathrm{v})$ agarose gel stained with GelRed ${ }^{\mathrm{TM}}$ while the remainder was sequenced in an ABI3730XI DNA Analyser (Beijing Tsingke Biotechnology Co., Beijing, PR China).

DNA sequences were viewed and manually corrected for any misread nucleotide and aligned using BioEdit software [21]. The identity of each isolate was confirmed in a BLAST search in the GenBank database using the NCBI BLAST algorithm (https://blast.ncbi.nlm.nih.gov/Blast.cgi). The diversity (nucleotide and haplotype diversity) and neutrality (Fu's Fs and Tajima's D) indices were estimated in DnaSP v.6 [22]. Bayesian phylogeny was inferred based on the nad 1 and cox 1 dataset using MrBayes v.3.1.2. Markov chain Monte Carlo (MCMC) sampling was used to assess the posterior distribution of the parameters with a chain length of 2,000,000 states, and $10 \%$ was discarded as burn-in. Parameters were logged every 1,000 states. TreeView v.1.6.6. (http://taxonomy.zoology. 

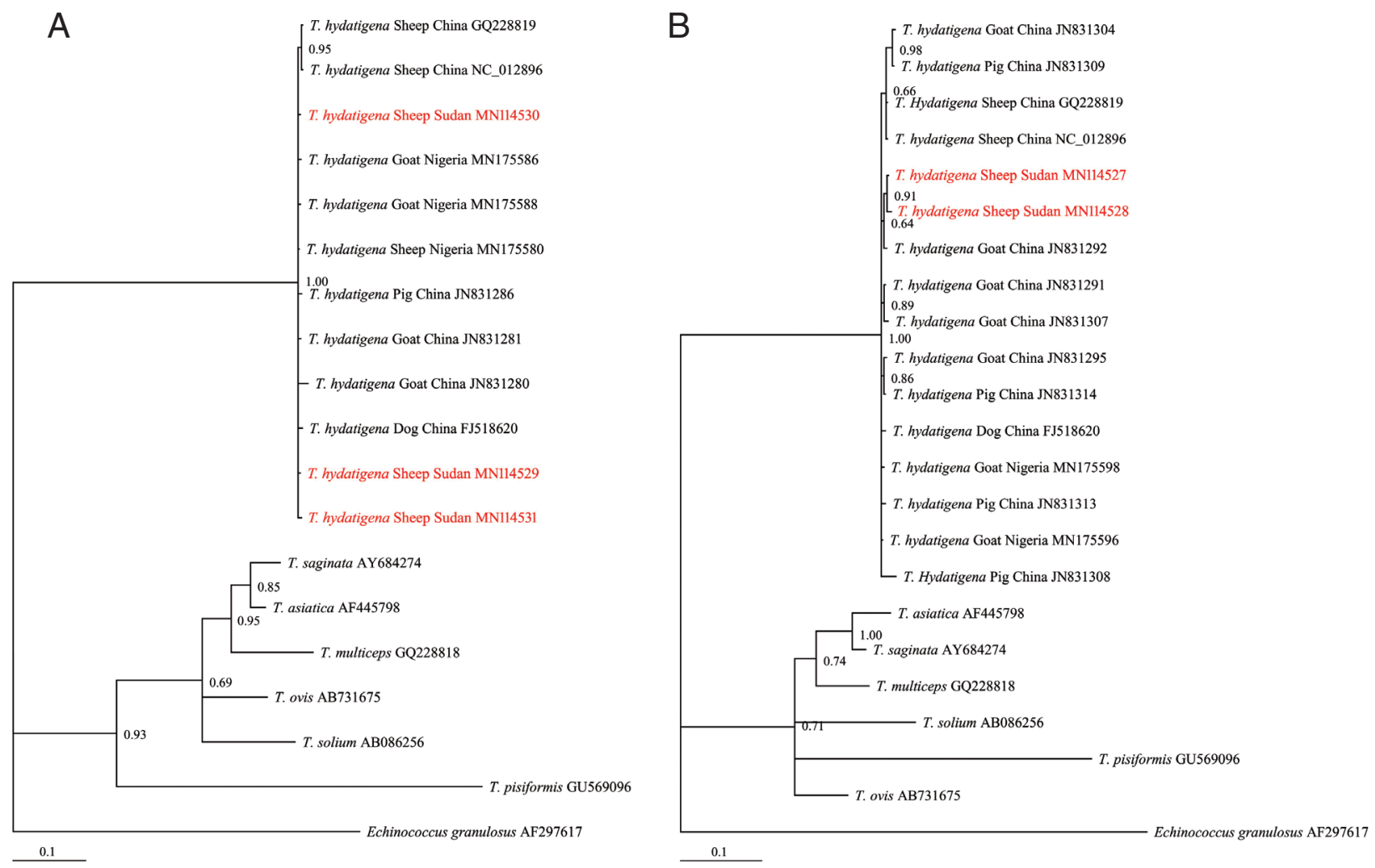

Fig. 2. Bayesian phylogenetic relationship of T. hydatigena isolates with other Taenia species. (A) nad1. (B) cox1. Red, Sudanese haplotype representing sheep isolates from this study.

gla.ac.uk/rod/treeview.html) was used to draw/display trees.

Of the 30 cyst samples, successful amplification and sequencing of the nad 1 and cox1 genes identified 11 cysts as $T$. hydatigena. No amplification was observed for the other 19 cysts, even after the process was repeated. The useable nucleotide sequences after correction and editing were 764 and 735 bp for nad 1 and cox1, respectively. These sequences were more than $99 \%$ similar to those in the GenBank repository. The Sudanese T. hydatigena isolates showed the highest similarity (99.73-99.87\%) to the Chinese and Nigerian isolates according to the BLAST query. Due to availability of partial nad 1 and cox1 genes from different geographical locations, phylogenetic analysis was conducted using 677 and 735 bp of the nad 1 and cox1 genes, respectively. The Bayesian phylogeny further confirmed identity of the isolates, as they clustered with other reference $T$. hydatigena sequences from the GenBank database (Supplementary Table S1) and exhibited significant distances from other Taenia species (T. saginata, T. ovis, T. pisiformis, T. solium, T. multiceps, and T. asiatica) (Fig. 2A, B), indicating that all isolates were clearly $T$. hydatigena. This result was also con-
Table 1. Diversity and neutrality indices of a Taenia hydatigena population from Sudan based on partial nad1 (764 bp) and cox1 genes (735 bp)

\begin{tabular}{lcc}
\hline Index & nad1 & cox1 \\
\hline No. of isolates & 11 & 9 \\
No. of mutations & 4 & 2 \\
Parsimony informative site & 1 & 0 \\
No. of haplotypes & 3 & 2 \\
Haplotype diversity (Hd) & $0.345 \pm 0.172$ & $0.222 \pm 0.166$ \\
Nucleotide diversity $(\pi)$ & $0.00114 \pm 0.00059$ & $0.00060 \pm 0.00045$ \\
Tajima's D & -1.32167 & -1.3624 \\
Fu's Fs & 0.323 & 0.671 \\
\hline
\end{tabular}

firmed by the posterior probability (PP) values between the isolates, although a few had PP values $<0.70$. This low nodal support value between $T$. hydatigena species was also recently reported in Nigeria [18]. The nucleotide sequences of cox 1 and nad 1 genes representing $T$. hydatigena isolates from the present study were deposited in GenBank under the following accession numbers (cox1: MN114527 and MN114528; nad1: MN 114529-MN114531). 
Analysis on the nad1 (764 bp) and cox1 (735 bp) mitochondrial gene sequences revealed 4 (1 parsimony informative) and 2 mutations (not parsimony informative), respectively. No deletions or insertions were observed. Low haplotype (Hd) and nucleotide $(\pi$ ) diversities were observed (Table 1 ). Regardless of small sample size, the observed nad1 Hd was comparable to $T$. hydatigena populations reported in dogs and goats, although with a lower $\pi$ compared to the T. hydatigena populations from the same study [29]. A comparison of the cox 1 diversity indices also revealed lower $\mathrm{Hd}$ and $\pi$ than the populations from Italian, Iranian, and Palestinian sheep [29]. Meanwhile, comparable data from an African host on the diversity indices of $T$. hydatigena are lacking $[4,17]$. However, compared with the recent report from Nigeria [18], the Sudanese T. $h y$ datigena isolates showed lower genetic variation.

Based on the nad 1 gene, 3 haplotypes were observed, with the nSDN1 haplotype constituting $81.8 \%$ (9/11) of the population. All haplotypes were separated from each other by no more than a 3-point mutational difference. The number of haplotypes based on the cox 1 gene was lower compared to the number of haplotypes (4) reported among a T. hydatigena population of Tanzanian sheep and goats [4] but similar to the number of haplotypes reported in Nigerian sheep [18]. The comparison of the nucleotide sequences from this study with the deposited sequences in the GenBank database yielded no haplotype with $100 \%$ identity, which could suggest the presence of unique haplotypes in Sudan and is consistent with previous reports of the existence of geographically distinct species of $T$. hydatigena [29]. Nonetheless, with limited sample size and the use of short mitochondrial DNA (mtDNA) fragments, further investigation would be necessary to understand and clearly describe the genetic differences and relationships between Sudanese T. hydatigena isolates and those from different geographical regions. Furthermore, we observed negative insignificant values of Tajima's $D$ and insignificant positive Fu's Fs values (Table 1), indicating inconsistencies with population expansion or a non-significant deviation from neutrality. As expected, although the low sample size may not reflect the actual status of the parasite population in Sudan, it provides a clue to the genetic status of the T. hydatigena population in the country.

The genetic ecology of most parasites of medical and veterinary importance has been found to contribute to understanding the epidemiology and control of parasitic infections. In this study, the results suggest some degree of genetic variation among Sudanese T. hydatigena isolates based on partial nad 1 and cox 1 mitochondrial genes. While the isolates were highly similar to isolates from other locations, no $100 \%$ match was observed. Globally, there still is a dearth of information on the genetic variation of $T$. hydatigena species, and with few available studies from Africa, this report contributes significant preliminary data on the genetic variation of $T$. hydatigena species in Sudan. Finally, we recommend that molecular investigations that include large sample sizes and utilize complete mitochondrial gene markers in addition to the nad 1 and cox 1 genes be considered in the future to provide further insight regarding the genetic variation and population structure of T. $h y$ datigena in Sudan.

\section{ACKNOWLEDGMENTS}

This study was supported by the National Key Research and Development Plan (2017YFD0501301, 2017YFD0501302), the Central Public-interest Scientific Institution Basal Research Fund (1610312016012, 1610312017001) and innovation fund 1 of the Chinese Academy of Agricultural Sciences (CAAS), while a scholarship was provided by the Chinese Government Scholarship (CGS) Programme of the Peoples Republic of China for Rosline James Muku.

\section{CONFLICT OF INTEREST}

The authors declare no conflict of interest related to this study.

\section{REFERENCES}

1. Kusiluka L, Kambarage D, Harrison L, Daborn C, Matthewman R. Causes of morbidity and mortality in goats in Morogoro district, Tanzania: The influence of management. Small Rumin Res 1998; 29: 167-172.

2. Christodoulopoulos G, Theodoropoulos G, Petrakos G. Epidemiological survey of cestode-larva disease in Greek sheep flocks. Vet Parasitol 2008; 153: 368-373.

3. Saulawa M, Magaji A, Faleke O, Mohammed A, Kudi A, Musawa A, Sada A, Ugboma A, Akawu B, Sidi S. Prevalence of Cysticercus tenuicollis cysts in sheep slaughtered at Sokoto abattoir, Sokoto state, Nigeria. Sokoto J Vet Sci 2011; 9: 24-27.

4. Braae UC, Kabululu M, Nørmark ME, Nejsum P, Ngowi HA, Johansen MV. Taenia hydatigena cysticercosis in slaughtered pigs, goats, and sheep in Tanzania. Trop Anim Health Prod 2015; 47: 1523-1530. 
5. Fakae B. The epidemiology of helminthosis in small ruminants under the traditional husbandry system in eastern Nigeria. Vet Res Commun 1990; 14: 381-391.

6. Sissay MM, Uggla A, Waller PJ. Prevalence and seasonal incidence of larval and adult cestode infections of sheep and goats in eastern Ethiopia. Trop Anim Health Prod 2008; 40: 387-394.

7. Belem A, Kabore A, Bessin R. Gastrointestinal helminthes of sheep in the central, eastern and northern part of Burkina Faso. Bull Anim Health Prod Afr 2005; 53: 13-23.

8. Attindehou S, Salifou S. Epidemiology of cestodes infections in sheep and goats in Benin. Vet Res 2012; 5: 59-62.

9. El Badawi KS, El Gezuli AY, Eisa AM, Slepnev NK. Incidence of Cysticercus tenuicollis in animals slaughtered for human consumption in Sudan. Sudan J Vet Sci Anim Husb 1978; 19: 87-91.

10. Bowles J, Blair D, McManus DP. Genetic variants within the genus Echinococcus identified by mitochondrial DNA sequencing. Mol Biochem Parasitol 1992; 54: 165-174.

11. Lavikainen A, Lehtinen MJ, Meri T, Hirvelä-Koski V, Meri S. Molecular genetic characterization of the Fennoscandian cervid strain, a new genotypic group (G10) of Echinococcus granulosus. Parasitology 2003; 127: 207-215.

12. Nakao M, Li T, Han X, Ma X, Xiao N, Qiu J, Wang H, Yanagida T, Mamuti W, Wen H, Moro PL, Giraudoux P, Craig PS, Ito A. Genetic polymorphisms of Echinococcus tapeworms in China as determined by mitochondrial and nuclear DNA sequences. Int J Parasitol 2010; 40: 379-385.

13. Ito A, Yamasaki H, Nakao $M$, Sako $Y$, Okamoto $M$, Sato $M O$, Nakaya K, Margono SS, Ikejima T, Kassuku AA, Afonso SMS, Ortiz WB, Plancarte A, Zoli A, Geerts S, Craig PS. Multiple genotypes of Taenia solium ramifications for diagnosis, treatment and control. Acta Trop 2003; 87: 95-101.

14. Zhang Y, Zhao W, Yang D, Tian Y, Zhang W, Liu A. Genetic characterization of three mitochondrial gene sequences of goat/ sheep-derived Coenurus cerebralis and Cysticercus tenuicollis isolates in Inner Mongolia, China. Parasite 2018; 25: 1.

15. Maravilla P, Gonzalez-Guzman R, Zuniga G, Peniche A, Dominguez-Alpizar JL, Reyes-Montes R, Flisser A. Genetic polymorphism in Taenia solium cysticerci recovered from experimental infections in pigs. Infect Genet Evol 2008; 8: 213-216.

16. Vega R, Pinero D, Ramanankandrasana B, Dumas M, Bouteille B, Fleury A, Sciutto E, Larralde C, Fragoso G. Population genetic structure of Taenia solium from Madagascar and Mexico: implications for clinical profile diversity and immunological technology. Int J Parasitol 2003; 33: 1479-1485.

17. Omar MA, Elmajdoub LO, Al-Aboody MS, Elsify AM, Elkhtam AO, Hussien AA. Molecular characterization of Cysticercus tenuicollis of slaughtered livestock in Upper Egypt governorates. Asian Pac J Trop Biomed 2016; 6: 706-708.
18. Ohiolei JA, Luka J, Zhu GQ, Yan HB, Li L, Magaji AA, Alvi MA, Wu YT, Li JQ, Fu BQ, Jia WJ. First molecular description, phylogeny, and genetic variation of Taenia hydatigena from Nigerian sheep and goats based on three mitochondrial genes. Parasit Vectors 2019; 12: 520.

19. Jia WZ, Yan HB, Guo AJ, Zhu XQ, Wang YC, Shi WG, Chen HT, Zhan F, Zhang SH, Fu BQ, Littlewood DT, Cai XP. Complete mitochondrial genomes of Taenia multiceps, T. hydatigena and T. pisiformis: additional molecular markers for a tapeworm genus of human and animal health significance. BMC Genomics 2010; 11: 447.

20. Wu Y, Li L, Zhu G, Li W, Zhang N, Li S, Yao G, Tian W, Fu B, Yin H, Zhu X, Yan H, Jia W. Mitochondrial genome data confirm that yaks can serve as the intermediate host of Echinococcus canadensis (G10) on the Tibetan Plateau. Parasit Vectors. 2018; 11: 166.

21. Hall TA. BioEdit: a user-friendly biological sequence alignment editor and analysis program for Windows 95/98/NT. Nucl Acids Symp Ser 1999; 41: 95-98.

22. Rozas J, Ferrer-Mata A, Sanchez-DelBarrio JC, Guirao-Librado P, Remos-Onsins SE, Sanchez-Gracia A. DnaSP 6: DNA sequence polymorphism analysis of large data set. Mol Biol Evol 2017; 34: 3299-3302.

23. Liu GH, Lin RQ, Li MW, Liu W, Liu Y, Yuan ZG, Song HQ, Zhao $\mathrm{GH}$, Zhang KX, Zhu XQ. The complete mitochondrial genomes of three cestode species of Taenia infecting animals and humans. Mol Biol Rep 2011; 38: 2249-2256.

24. Jeon HK, Kim KH, Eom KS. Complete sequence of the mitochondrial genome of Taenia saginata: comparison with T. solium and T. asiatica. Parasitol Int 2007; 56: 243-246.

25. Jeon HK, Eom KS. Taenia asiatica and Taenia saginata: genetic divergence estimated from their mitochondrial genomes. Exp Parasitol 2006; 113: 58-61.

26. Nakao M, Lavikainen A, Iwaki T, Haukisalmi V, Konyaev S, Oku Y, Okamoto M, Ito A. Molecular phylogeny of the genus Taenia (Cestoda: Taeniidae): Proposals for the resurrection of Hydatigera Lamarck, 1816 and the creation of a new genus Versteria. Int J Parasitol 2013; 43: 427-437.

27. Nakao M, Sako Y, Ito A. The Mitochondrial Genome of the Tapeworm Taenia solium: a finding of the abbreviated stop codon U. J Parasitol 2003; 89: 633-635.

28. Le TH, Pearson MS, Blair D, Dai N, Zhang LH, McManus DP. Complete mitochondrial genomes confirm the distinctiveness of the horse-dog and sheep-dog strains of Echinococcus granulosus. Parasitology 2002; 124: 97-112.

29. Boufana B, Scala A, Lahmar S, Pointing S, Craig PS, Dessì G, Zidda A, Pipia AP, Varcasia A. A preliminary investigation into the genetic variation and population structure of Taenia hydatigena from Sardinia, Italy. Vet Parasitol 2015; 214: 67-74. 


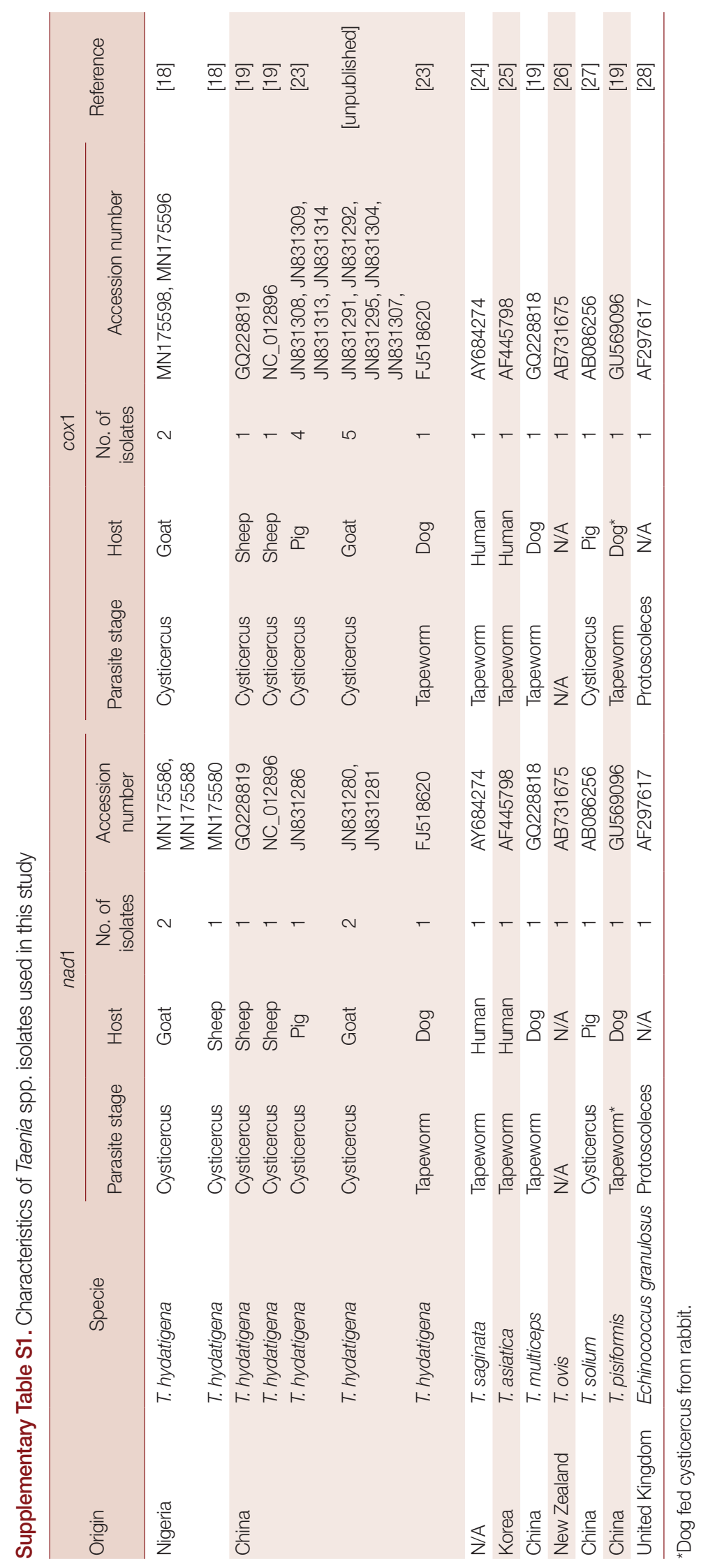

